\title{
LỰA CHỌN CHƯƠNG TRÌNH VÀ SỬ DỰNG KẾT QUẢ THAM GIA THỬ NGHIỆM THÀNH THẠO TRONG KIỂM NGHIẸM THỰC PHẨM
}

\author{
Nguyễn Thị Ngọc Thùy ${ }^{1}$, Hồ Trần Ngọc Quyên, Luơng Thanh Uyên \\ Trung tâm Kỹ thuật Tiêu chuẩn Đo luờng Chất lượng 3
}

(Ngày đến tòa soạn: 12/6/2018; Ngày sủa bài sau phản biện: 16/7/2018; Ngày chấp nhận đăng: 25/7/2018)

\section{Tóm tắt}

THỦ'nghiệm thành thạo (TNTT) là một công cu khách quan để đánh giá năng lực của phòng 1 thi nghiệm (PTN). Tham gia TNTT giúp các PTN có thể tụ theo dõi và tư đánh giá độ tin cậy về kết quả phân tích của mình. Ngày nay, cùng với sự phát triển của các PTN, nhu cầu tham gia TNTT ngày càng tăng. Để đáp ưng nhu cầu này, hiện nay, số luọng đơn vị cung cấp và chwơng trình TNTT cũng trở nên phong phú và đa dạng hơn. Vì vậy, nếu không xây dựng các tiêu chí để lụa chọn chuoong trình TNTT và xác định rõ mục đích tham gia TNTT thì PTN có thể tốn kém rất nhiều thời gian, chi phi và nỗ lục vào hoạt động TNTT, đặc biệt đối với các PTN thưc hiện nhiều phép thư. Bài viết này sẽ cung cấp một số gợi ý cho việc lụa chọn chưong trình TNTT phù hợp và sủ dụng kết quả tham gia TNTT một cách hiệu quả đối với các PTN đặc biệt là các PTN trong lĩnh vực kiểm nghiệm thực phẩm.

Tù̀ khóa: Thử nghiệm thành thạo, kết quả kiểm nghiệm, kiểm nghiệm thực phẩm, lựa chọn chuoong trình TNTT, sử dụng kết quả TNTT.

\section{1. ĐẠT VẤN ĐỀ}

Thử nghiệm thành thạo (TNTT) là hoạt động nhằm đánh giá việc thực hiện các phép thử trên cùng một mẫu hoặc trên các mẫu tương đương nhau theo các điều kiện đã định trước giữa hai hay nhiều phòng thí nghiệm (PTN) [1]. Nói cách khác, TNTT đánh giá độ chính xác về kết quả phân tích bằng cách so sánh với một giá trị ấn định, được xem như là giá trị đúng của mẫu.

Hiện nay, theo các điều khoản của ISO/IEC 17025:2017 [2] và chính sách của các đơn vị công nhận PTN như BoA, A2LA, ILAC thì tham gia TNTT, so sánh liên phòng là một yêu cầu bắt buộc để công nhận và duy trì tình trạng công nhận phù hợp theo ISO/IEC 17025 của các PTN [3], [4], [5]. Phiên bản ISO/IEC 17025:2017 đặt ra thêm một yêu cầu mới cho các PTN là phải xây dựng một kế hoạch tham gia TNTT cho PTN của mình (Mục 7.7.2) [2]).

Bài viết này đưa ra một số nguyên tắc cho việc lựa chọn chương trình và đơn vị cung cấp chương trình TNTT phù hợp nhất khi xây dựng kế hoạch tham gia TNTT của PTN. Đồng thời cung cấp một số gợi ý cho việc sử dụng kết quả tham gia TNTT tại PTN và các đối tượng quan tâm nhằm giúp các PTN tiết kiệm thời gian, chi phí và nỗ lực vào việc tham gia TNTT. Để đảm bảo tính cụ thể và tính

${ }^{1}$ Điện thoại:0986758002 Email:qt-tntt@quatest3.com.vn 
khả thi khi áp dụng vào thực tế, phạm vi bài viết chỉ giới hạn trong lĩnh vực kiểm nghiệm thực phẩm. 2. LỬA CHỌN ĐƠN VỊ TỔ CHỨC VÀ CHƯỚNG TRÌNH THỬ NGHIỆM THÀNH THẠO

Tham gia TNTT là một phương pháp khách quan để đánh giá và chứng minh độ tin cậy của kết quả thử nghiệm (KQTN) cũng như đo lường năng lực của PTN bên cạnh các công cụ kiểm soát chất lượng nội bộ. Vì vậy, tất cả các PTN cần đưa ra một kế hoạch tham gia TNTT phù hợp với hoàn cảnh của mình [6].

Các yếu tố cần xem xét trong một kế hoạch tham gia TNTT bao gồm: chương trình TNTT, đơn vị tổ chức và tần suất tham gia.

\subsection{Lựa chọn đơn vị tổ chức TNTT}

Để kết quả tham gia TNTT có độ tin cậy cao, PTN cần phải lựa chọn các đơn vị tổ chức đủ năng lực. Khi lựa chọn, PTN nên ưu tiên xem xét các khía cạnh sau:

Thứ nhất, đơn vị tổ chức đã được công nhận phù hợp theo tiêu chuẩn ISO/IEC 17043 hay chưa? Mặc dù ISO 17025:2017 chỉ yêu cầu đơn vị tổ chức TNTT tuân theo ISO/IEC 17043, việc đã được công nhận sẽ đảm bảo các chương trình TNTT luôn được đặt dưới sự giám sát của một bên thứ ba. Tham gia chương trình TNTT của đơn vị đã được công nhận là một trong những khuyến nghị của các cơ quan công nhận PTN như ILAC, A2LA [4], [5].

Thứ hai, ưu tiên sử dụng các đơn vị có kinh nghiệm tổ chức lâu năm bởi kinh nghiệm tổ chức rất quan trọng trong việc đánh giá kết quả của các PTN, đảm bảo độ đồng nhất và ổn định của mẫu thử. Điều này còn đảm bảo chương trình được diễn ra theo đúng thời gian quy định, tránh được các trường hợp hủy hoặc hoãn chương trình do các sự cố kỹ thuật.

Thứ ba, phương thức xử lý số liệu ảnh hưởng rất lớn đến kết quả tham gia TNTT của các PTN. Mặt khác, với mỗi mục đích tham gia TNTT khác nhau cần những phương thức xử lý số liệu khác nhau. Vì vậy, PTN cần phải tìm hiểu và biết trước phương thức xử lý thống kê đối với các chương trình dự định tham gia. PTN nên ưu tiên lựa chọn đơn vị tổ chức có phương thức xử lý số liệu và đánh giá kết quả tham gia TNTT rõ ràng, minh bạch và dễ tiếp cận.

Thứ tư, trong TNTT bảo mật thông tin của PTN là vô cùng quan trọng để tránh các bên thứ ba sử dụng kết quả TNTT của mình với những mục đích không mong muốn, vì vậy đơn vị có chính sách và biện pháp bảo mật thông tin chặt chẽ nên được ưu tiên lựa chọn.

Thứ năm, PTN cũng không nên bỏ qua việc xem xét về sự thuận tiện trong việc tương tác giữa đơn vị tổ chức và $\mathrm{PTN}$ tham gia dựa vào các yếu tố như: ngôn ngữ sử dụng, sự rõ ràng và dễ tiếp cận thông tin, tiến độ chương trình, mức độ hỗ trợ và đáp ứng yêu cầu của $\mathrm{PTN}$ về TNTT [7] .

Ngoài ra, các PTN xin công nhận ở cơ quan công nhận nào thì nên lựa chọn các đơn vị tổ chức nằm trong danh sách nhà cung cấp TNTT được chấp nhận của cơ quan công nhận đó.

\subsection{Lựa chọn chương trình TNTT}

Hiểu theo cách đơn giản nhất, chương trình TNTT phù hợp là chương trình có nền mẫu, chỉ tiêu và nồng độ hoạt chất hoàn toàn tương đồng với mẫu thông thường của PTN. Thực tế, PTN, đặc biệt là các $\mathrm{PTN}$ có mẫu thử đặc thù, rất khó tìm kiếm các chương trình TNTT hoàn toàn tương đồng vì sự hạn chế về số lượng PTN có thể dẫn đến bộ dữ liệu quá nhỏ và kết quả TNTT không tin cậy.

Eurachem cũng đưa ra đề xuất là trong trường hợp chương trình TNTT lý tưởng cho yêu cầu của PTN không có sẵn, PTN có thể lựa chọn các chương trình tương tự thay thế [8].

Ở đây, khái niệm "phù hợp" nên được mở rộng dựa trên các tiêu chí sau:

- Phạm vi áp dụng của phương pháp thử. Ví dụ các phương pháp thử áp dụng cho cả thực phẩm 
và thức ăn chăn nuôi thì PTN có thể lựa chọn tham gia một trong hai loại nền mẫu này,

- Sự tương đồng về bản chất giữa các loại nền mẫu, như giữa sữa bột và bột dinh dưỡng,

- Đánh giá sự ảnh hưởng của nền mẫu đối với kết quả phân tích, trường hợp nền mẫu không ảnh hưởng nhiều đến kết quả thì có thể lựa chọn nền mẫu mở rộng hơn. Ví dụ các nền mẫu thực phẩm thông thường như sữa bột, ngũ cốc, bột dinh dưỡng thì nền mẫu có ảnh hưởng không đáng kể đến kết quả phân tích các chỉ tiêu vi sinh cơ bản như TPC, Coliforms, E.coli, men mốc nên PTN có thể lựa chọn tham gia một trong các loại nền mẫu này.

\subsection{Mức độ và tần suất tham gia TNTT}

Về mức độ tham gia TNTT, lý tưởng nhất là ứng với mỗi chỉ tiêu cho mỗi loại sản phẩm, PTN sẽ tham gia một chương trình TNTT riêng biệt. Tuy nhiên, điều này là không khả thi cả về tính logic và tính kinh tế. Vì vậy, các cơ quan công nhận chỉ nên yêu cầu các PTN tham gia TNTT theo từng nhóm dựa trên kỹ thuật phân tích, bản chất mẫu thử sao cho kết quả tham gia TNTT này có thể đại diện cho các kỹ thuật phân tích, thuộc tính của cả nhóm [9]. Tuy nhiên, sự phân nhóm giữa các PTN có thể khác nhau, tùy thuộc vào sự giải thích về tính hợp lý trong cách phân nhóm của PTN [9].

Về tần suất tham gia TNTT, mỗi PTN khác nhau sẽ có tần suất tham gia TNTT khác nhau. Tần suất này được xây dựng dựa trên sự xem xét mức độ áp dụng các biện pháp đảm bảo chất lượng khác [9]. Tần suất cũng có thể thay đổi khi đã xác minh được độ tin cậy của các biện pháp đảm bảo chất lượng nội bộ, có thể giãn tần suất tham gia so với ban đầu trong trường hợp các biện pháp kiểm soát nội bộ có độ tin cậy cao. Mặt khác, tần suất tham gia TNTT tối thiểu phải đáp ứng được yêu cầu của cơ quan công nhận như: BoA Việt Nam yêu cầu phải có kết quả tham gia TNTT trước khi đánh giá và ít nhất 3 năm/lần đối với từng lĩnh vực [3], A2LA yêu cầu ít nhất 2 lần/năm [5] .

\section{SỬ DỤNG KẾT QUẢ THỬ NGHIẸM THÀNH THẠO}

Kết quả TNTT là sự đánh giá khách quan và tin cậy về năng lực kỹ thuật của PTN. Tham gia TNTT theo đó sẽ đóng một vai trò quan trọng trong việc quản lý đối với bản thân PTN, các đơn vị chủ quản, cơ quan nhà nước và các tổ chức công nhận PTN nếu kết quả TNTT được sử dụng một cách đúng đắn và hiệu quả. Dưới đây là một số gợi ý về việc sử dụng kết quả TNTT:

\section{1. Đối với PTN}

\subsubsection{Chứng minh năng lực kỹ thuật}

PTN có thể sử dụng kết quả tham gia TNTT tốt như một bằng chứng khách quan, thuyết phục để khẳng định năng lực thử nghiệm trong các trường hợp tranh chấp, nghi ngờ về kết quả phân tích cũng như cam kết các vấn đề về chất lượng.

\subsubsection{Công cu quản lý mối nguy và cải tiến chất lương của PTN}

Kết quả tham gia TNTT được xem như công cụ quản lý chất lượng PTN theo các cách như sau:

- Thứ nhất, các kết quả TNTT không đạt sẽ được sử dụng như một yêu cầu về việc điều tra nguyên nhân gây sai số. Vì khi có kết quả TNTT không đạt, PTN được khuyến cáo tiến hành điều tra về các nguồn gây sai số tiềm ẩn. Khi không tham gia TNTT, các nguồn gây sai số này có thể không được phát hiện và PTN sẽ không đảm bảo có những hành động khắc phục kịp thời. Nó có thể dẫn đến việc tiếp tục cung cấp các kết quả thiếu chính xác cho khách hàng và cuối cùng có thể dẫn đến làm mất danh tiếng hoặc phải chịu các bất lợi khác từ pháp luật và các bên liên quan. Vì vậy, đây được xem như là một công cụ để quản lý mối nguy và cải tiến chất lượng của PTN [10]. 
- Thứ hai, tham gia chương trình TNTT để kiểm tra tính chính xác của phương pháp thử trước khi được áp dụng rộng rãi tại $\mathrm{PTN}$ là một cách khách quan, hiệu quả, ít tốn chi phí và công sức để xác nhận giá trị sử dụng của phương pháp thử.

- Thứ ba, mẫu TNTT có bản chất ổn định lâu dài, có thể được sử dụng như các mẫu đối chứng để kiểm soát chất lượng nội bộ mẫu sau khi chương trình đã kết thúc [10].

Ngoài ra, báo cáo kết thúc chương trình TNTT tổng hợp đầy đủ KQTN, độ không đảm bảo đo (ĐKĐBĐ), các phương pháp thử của các $\mathrm{PTN}$ tham gia... là một nguồn tài liệu tham khảo hữu ích cho việc đào tạo và cải tiến tay nghề của kiểm nghiệm viên.

\subsubsection{Tài liệu tham khảo để cải tiến quy trình phân tỉch}

Thông thường, báo cáo kết thúc các chương trình TNTT sẽ liệt kê đầy đủ các phương pháp thử của các $\mathrm{PTN}$ tham gia. $\mathrm{PTN}$ có thể sử dụng báo cáo này như là tài liệu tham khảo để so sánh kết quả phân tích giữa các phương pháp thử với nhau, lựa chọn phương pháp phù hợp hơn (nếu cần). Đồng thời, đây cũng là kênh thông tin cho các PTN cập nhật phiên bản mới nhất của phương pháp thử, kỹ thuật phân tích mới, hiện đại để cải tiến quy trình phân tích của mình.

\subsubsection{Xác định và kiểm tra độ không đảm bảo đo (ĐKĐBĐ) của phuoong pháp thử}

PTN có thể sử dụng kết quả TNTT để kiểm tra ĐKĐBĐ của mình theo cách sau: Thứ nhất, thông thường $\mathrm{DK} Đ \mathrm{~B}$ của một $\mathrm{PTN}$ phải tương xứng với độ rộng tất cả các kết quả tham gia TNTT của PTN đó trong một chương trình TNTT nhiều vòng [6]. Thứ hai, ĐKĐBĐ chuẩn trongmột kết quả phân tích sẽ được mong đợi thấp hơn độ tái lập trong chương trình TNTT. Nhưng nếu ĐKĐBĐ này thấp hơn quá nhiều thì phải xem lại việc đánh giá ĐКĐBĐ [11].

Mặt khác, đối với các chương trình TNTT sử dụng cùng một phương pháp thử thì độ lệch chuẩn giữa các PTN tham gia tương đương với độ lệch chuẩn tái lập của từng PTN, trải qua một số vòng nhất định, từ sự chênh lệch giữa các kết quả của PTN và giá trị ấn định, $\mathrm{PTN}$ có thể tự đánh giá sơ bộ ĐKĐBĐ trong phép thử [12].

\section{2. Đối với các bên quan tâm khác}

\subsubsection{Các co quan công nhận PTN}

Các PTN đạt kết quả tốt trong các chương trình TNTT là một trong những bằng chứng thuyết phục giúp các chuyên gia đánh giá tự tin khi chấp thuận dữ liệu PTN cung cấp để xin công nhận hoặc duy trì sự công nhận [6].

\subsubsection{Các đơn vị chủ quản PTN, co quan quản lý nhà nuớc}

Hiện nay, tại Việt Nam một số đơn vị chủ quản PTN và cơ quan quản lý nhà nước đã sử dụng kết quả tham gia TNTT trong việc chỉ định các PTN thực hiện các phép thử trong quản lý nhà nước và xem xét năng lực hiện tại của các PTN trực thuộc đơn vị mình.

\subsubsection{Khách hàng của PTN và các bên quan tâm khác}

Kết quả tham gia TNTT là một bằng chứng về năng lực kỹ thuật của PTN nên được sử dụng như là một trong các tiêu chí khi so sánh và lựa chọn dịch vụ thử nghiệm.

\section{KẾT LUẬN}

TNTT là công cụ quan trọng để đánh giá và chứng minh độ tin cậy của kết quả thử nghiệm (KQTN) cũng như đo lường năng lực của PTN bên cạnh các công cụ kiểm soát chất lượng nội bộ. Tuy nhiên, để công cụ này đạt được hiệu quả cao, trước hết, PTN cần xây dựng được một kế hoạch tham gia TNTT cụ thể, rõ ràng và phù hợp với tình hình thực tế. Hi vọng những gợi 
ý từ bài viết này có thể giúp các $\mathrm{PTN}$, đặc biệt là các $\mathrm{PTN}$ trong lĩnh vực kiểm nghiệm thực phẩm, dễ dàng hơn trong việc lập kế hoạch tham gia TNTT của đơn vị mình.

\title{
TÀI LIỆU THAM KHẢO
}

1. ISO 17043:2010, Conformity assessment. General requirements for proficiency testing.

2. ISO/IEC 17025:2017, General requirements for the competence of testing and calibration laboratories.

3. APL 03, Văn phòng công nhận chất lượng Việt Nam, (2016), Chính sách về Thử nghiệm thành thạo/So sánh liên phòng.

4. ILAC-P9:11, (2016), ILAC policy for participation in Proficiency testing activities.

5. R103, American Association for Laboratory Accreditation, (2013), General requirements: Proficiency tessing for ISO/IEC 17025 laboratories.

6. Brian Brookman Ian Mann, (2011), Selection, Use and Interpretation of Proficiency testing (PT) Schemes.

7. Eurachem's Proficiency testing Working Group, (2015), Selecting the right Proficiency testing scheme for my laboratory.

8. EUROLAB, (2017), Criteria for the selection of a Proficiency testing scheme, EUROLAB"Cook Book" - Doc No.2.

9. EA-4/18, European co-operation for Accreditation, (2010), Guidance on the level and frequency of proficiency testing participation.

10. ILAC Brochure, (2008), Benefits for laboratories participating in proficiency testing programs.

11. Eurachem's Proficiency testing Working Group, (2013), How can Proficiency testing help my labrotory.

12. EUROLAB, (2017), Measurement uncertainty revisited: Alternative approaches to uncertainty evaluation, p. 10-13.

\section{Summary}

SELECTING PROFICIENCY TESTING SCHEMES AND USING ITS RESULTS IN FOOD TESTING

\author{
Nguyen Thi Ngoc Thuy, Ho Tran Ngoc Quyen, Luong Thanh Uyen \\ Quality Assurance and Testing Center 3
}

Proficiency testing (PT) is an objective tool to assess the laboratory's performance. Participation in PT helps laboratories self-monitoring and self-evaluating the reliability of results. Nowadays, together with the development of laboratories, the demand of participating PT schemes is increasing, and there are variety of PT providers and PT schemes correspondingly. Hence, without of appropriate criteria for selection of $P T$ schemes and the purposes of participating PT, time, money and effort could be excessively consumed, especially for laboratories that perform numerous different tests. In this study, principles for choosing appropriate PT schemes and using the PT results effectively for laboratories, particularly in food testing laboratories were provided.

Keywords: Proficiency testing, testing result, food testing, selection of PT scheme, use of PT result. 